\title{
Spatial Screening of Lectin Ligands. Cyclic Peptides as Scaffolds for Multivalent Presentation of Carbohydrates
}

\author{
Valentin Wittmann and Sonja Seeberger \\ Institut für Organische Chemie, Johann Wolfgang Goethe-Universität, 60439 Frankfurt, \\ Germany
}

\section{Introduction}

Carbohydrate-lectin interactions are the basis of numerous biologically important recognition processes [1]. Examples include the initiation of the inflammatory response, bacterial and viral pathogenesis, fertilization, and even protein folding. High-affinity lectin ligands are of considerable medicinal interest in the diagnosis, therapy, and prevention of conditions associated with these processes. One approach to arrive at such ligands is the design of small oligovalent carbohydrate derivatives (mini clusters) which can simultaneously bind to several binding sites of a single (oligomeric) lectin proximate in space and may be tailored to lectins with known 3D-structure [2] ("directed" multivalency). However, if the structure of the targeted lectin and thus the required orientation of the sugar epitopes of the mini clusters are unknown, an efficient procedure for the generation and screening of libraries of spatially diverse mini clusters is desirable. Here we present a new strategy for finding multivalent lectin ligands by spatial screening using libraries of cyclic peptides as scaffolds for multivalent presentation of carbohydrate epitopes $[3,4]$.

\section{Results and Discussion}

Our strategy comprises four steps: a) split-mix synthesis of a library of scaffold molecules with side chain amino groups in varying amounts and spatial orientation, b) attachment of several copies of a carbohydrate ligand to the amino groups, c) on-bead screening of the library for lectin-binding properties, and d) identification of potent ligands by single-bead analysis.

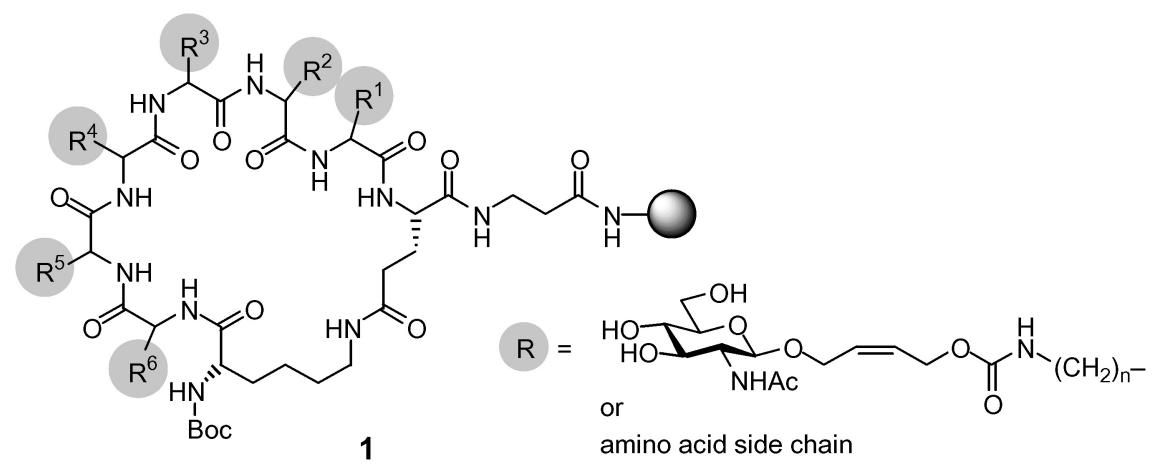

As scaffolds for the multivalent presentation of carbohydrate ligands, we have chosen cyclic peptides of general type $\mathbf{1}$. At the combinatorially varied positions indicated by gray circles, D- and L-amino acids without a side chain functionality as well as Dand L-diamino acids such as lysine, diaminobutyric acid, or diaminopropionic acid are incorporated. The latter represent the points of attachment of the carbohydrates. This library design allows for generation of spatial diversity in two dimensions. Positional diversity generates different carbohydrate patterns displayed on the scaffolds. Varying 
the stereochemistry of the amino acids increases spatial diversity by generating different backbone folds [5].

Application of this screening approach is demonstrated using wheat germ agglutinin (WGA) as an example. WGA, a $36 \mathrm{kDa}$ lectin composed of two glycine- and cysteine-rich subunits, contains several carbohydrate binding sites for $N$-acetylglucosamine (GlcNAc) and oligomers thereof, thus being a promising candidate for a multivalent interaction. Using a synthetic approach developed earlier in our laboratory [3], a neoglycopeptide library of 19440 compounds was synthesized on aminofunctionalized TentaGel without employing a linker following the "split and combine" synthesis method (Figure 1). GlcNAc residues were attached to side chain amino groups via an Aloc derived urethane. The carbohydrate content of the library members ranges from 0 (2.6\% of all compounds) over 1 (14.5\%), 2 (30.3\%), $3(30.9 \%), 4(16.6 \%), 5$ $(4.5 \%)$ up to $6(0.5 \%)$.
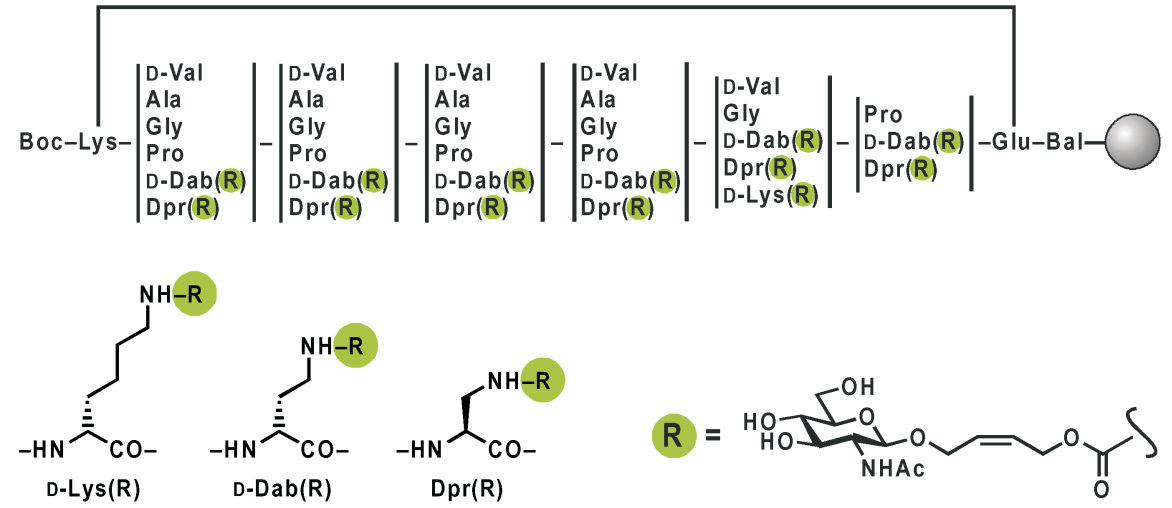

Fig. 1. Library of 19440 cyclic neoglycopeptides. Bal $=\beta$-alanine.

In order to screen the library for lectin binding properties, we developed an on-bead assay (Figure 2). Briefly, the resin-bound neoglycopeptides were incubated with biotinylated WGA followed by addition of an anti-biotin alkaline-phosphatase conjugate. Beads with bound lectin were detected by an alkaline phosphatase catalyzed color reaction. When the assay was carried out in the presence of competing monovalent ligand (GlcNAc), a small part (approx. 0.1\%) of the beads stained very darkly. These beads were manually selected under a microscope and treated with

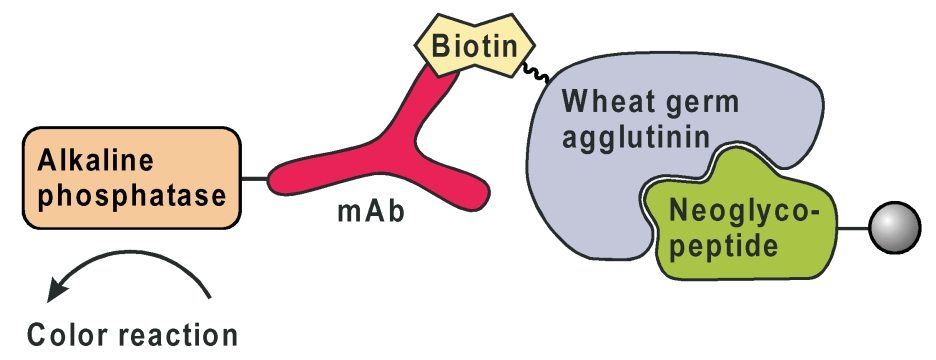

Fig. 2. Immunosorbent lectin binding assay. 
$\left[\mathrm{Pd}\left(\mathrm{PPh}_{3}\right)_{4}\right] /$ morpholine in order to remove the carbohydrates. After cleavage of the $N$-terminal Boc protecting group, "hit" structures were identified by automated single-bead Edman degradation. Due to the side chain cyclization, a negative answer is expected during the first degradation step, as long as cyclization has occurred completely (cyclization control).

So far, three WGA ligands could be identified, the hexavalent compounds $\mathbf{2}$ and $\mathbf{3}$ and, interestingly, tetravalent 4. Its four $\mathrm{D}-\mathrm{Dab}(\mathrm{R})$ residues in positions 2, 4, 5, and 7 are conserved among all three ligands. Except for position 3 in compound $2(\operatorname{Dpr}(\mathrm{R}))$, we found exclusively D-amino acids at the combinatorially varied positions $2-7$. Further sequencing results are necessary to confirm the observed consensus sequence and preference for D-amino acids.
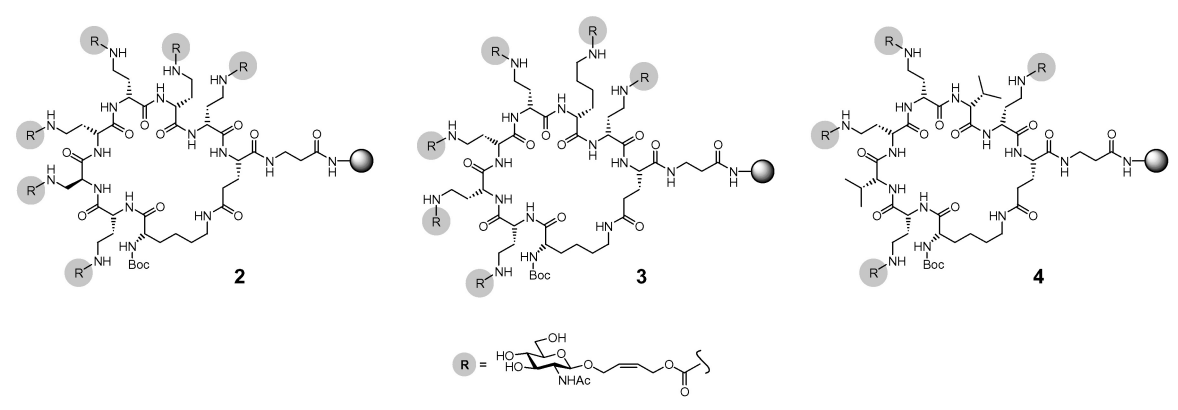

Since the corresponding non-glycosylated cyclopeptide library (Figure 1, R = Ac) does not contain any WGA ligands, we assume the spatial presentation of the GlcNAc residues on the cyclopeptide scaffolds to be responsible for different binding affinities to WGA.

\section{Acknowledgments}

This work was kindly supported by the Deutsche Forschungsgemeinschaft (grants WI 1479/2-1 and 2-2) and the Adolf Messer-Stiftung (Adolf Messer-Stiftungspreis 2000 for interdisciplinary research to V. W.).

\section{References}

1. Varki, A., Cummings, R., Esko, J., Freeze, H., Hart, G., Marth, J. (Eds.) Essentials of Glycobiology, Cold Spring Harbor Laboratory Press, Cold Spring Harbor, 1999.

2. a) Kitov, P.I., et al. Nature 403, 669-672 (2000); b) Fan, E., et al. J. Am. Chem. Soc. 122, 2663-2664 (2000); c) Mammen, M., et al. Angew. Chem., Int. Ed. 37, 2754-2794 (1998).

3. Wittmann, V., Seeberger, S. Angew. Chem., Int. Ed. 39, 4348-4352 (2000).

4. On the use of cyclic peptides as scaffolds see: a) Franzyk, H., et al. Bioorg. Med. Chem. 5, 21-40 (1997); b) Sprengard, U., et al. Angew. Chem., Int. Ed. Engl. 35, 321-324 (1996); c) Tuchscherer, G., Mutter, M. Pure Appl. Chem. 68, 2153-2162 (1996).

5. Haubner, R., Finsinger, D., Kessler, H. Angew. Chem., Int. Ed. Engl. 36, 1374-1389 (1997). 